\title{
Frequency of Transition of patients with NYHA class III / IV to NYHA class I / II / III of stage C and D Heart Failure with Pharmacotherapy in a Tertiary Level Hospital
}

SANJIDA ANSARI, MD. FAISAL IBN KABIR, KHURSHED AHMED, SYED ALI AHSAN, CHAUDHARY MESKAT AHMED, MD. FAKHRUL ISLAM KHALED, MAUSOOL SIRAJ, JANNATUN NAHAR, SUMON KUMAR BISWAS, RAJAN KARNA, MOHAMMAD HASHIMUL AHSAN, RASHEDUL ISLAM, MD. HARISUL HOQUE

Department of Cardiology, University Cardiac Center, Bangabandhu Sheikh Mujib Medical University (BSMMU), Dhaka, Bangladesh Address of Correspondence: Dr. Sanjida Ansari, Resident, Department of Cardiology, BSMMU, Dhaka E-mail: drsanjidaansari@gmail.com

\begin{abstract}
:
Background: Heart failure (HF) is one of the most important health problems in terms of prevalence, morbidity, mortality and health service use. It affects around 2 to $3 \%$ of the population. NYHA class can be used for the prioritization, triage and tailoring the HF management which is the foundation for the selection of therapies. The patient with higher NYHA class may need Mechanical Circulatory Support therapy or palliative care or hospice care. The identification of the patients with lower NYHA class helps to tailor vigorous drug therapy and close follow up program, the prognosis of these low risk patients maybe further improved. It is a simple tool for risk stratification in clinical practice.

Objective: The principal objective of this study was to determine frequency of transition of NYHA class III / IV to NYHA class I / II / III of stage C \& D HF with drugs in a tertiary level hospital. Methods: This was a crosssectional study. A total of 45 patients with stage $C$ and D HF were enrolled in the study by consecutive sampling from October 2019 to September 2020. Detailed history including NYHA functional class of stage C and D HF, physical examination, relevant investigations and Echocardiography were done in all the subjects. The subjects were treated accordingly. The treatment response was assessed again with NYHA functional class on discharge.

Result: Patients had mean age of $62 \& 60$ and 54 \& 54 years for NYHA class III \& IV of stage C and D HF. Majority of the patients were male. Primary cause of HF for both stage were IHD followed by DCM and valvular heart disease. The clinical presentation of stage $C \& D H F$ was improved significantly on discharge. Haematological, biochemical, radiological and echocardiographic findings of $\mathrm{NYHA}$ class IV of stage $C$ \& $D$ HF was more worst.

Conclusion: There is statistically significant transition of NYHA class III of stage C and D HF to lower NYHA class but there is no statistically significant transition of NYHA class IV of stage C and D HF with pharmacotherapy in a tertiary level hospital. Higher NYHA class is associated with poor outcome of stage C \& D HF patients.
\end{abstract}

Keywords: NYHA class III / IV of stage C and D, pharmacotherapy, transition to NYHA class I / II / III of stage $\mathrm{C}$ and $\mathrm{D} \mathrm{HF}$

University Heart Journal 2022; 18(1): 14-21

Introduction:

The health related quality of life of patients with HF is an important outcome as it reflects the impact of HF on their lives. ${ }^{1}$ The New York Heart Association (NYHA) classification is the most commonly used system to describe the impact of HF on a patient's daily activities. The classification was originally developed in 1928 and subsequently revised. It classifies the patients with HF into 4 categories (I, II, III and IV) with higher class indicating more severe symptoms, limitation in physical activity. Clinicians assign NYHA class on the basis of their indirect interpretation of reported patient's symptoms and medical history. Physician-assigned NYHA class is more predictive of outcome in HF patients including hospitalizations and mortality. ${ }^{2}$ NYHA class provides more reliable and clinically more useful prognostic information. It is a simple tool for risk stratification in clinical practice and can be used to help tailor management of HF. ${ }^{3}$ It targets the high risk population and help simplifying the difficult decision. It determines the clinical eligibility and candidacy for drugs and devices. ${ }^{4}$ The primary goal of classifying NYHA class is to help clinicians to integrate these concepts into routine practice to deliver effective patient centered care. Aging, comorbid condition, end organ damage, cognitive impairment, frailty, limited social support, accessibility and affordability in this part of the globe complicates the 
HF management. ${ }^{5}$ Thus NYHA class can be used for the prioritization, triage and tailoring the HF management which is the foundation for the selection of therapies. The patient with higher NYHA class may need device therapy or palliative care or hospice care. ${ }^{6}$ The identification of lower NYHA class helps to maximize the drug therapy and close follow up which improves the prognosis further. The aim of this study was to determine frequency of transition of NYHA class III / IV to NYHA class I / II/ III of stage $\mathrm{C}$ and D HF with drugs and may also serve as background work to conduct further research in Bangladesh. This study might help to understand the importance of NYHA class in clinical practice in resource constraint settings as a useful prognostic marker in HF patients.

\section{Materials and Methods}

Study population: This cross sectional study was conducted in department of Cardiology, Bangabandhu Sheikh Mujib Medical University, Dhaka from September, 2019 to August, 2020. A total of 45 cases with NYHA class III \& IV of stage C \& D HF diagnosis patients were enrolled in the study who were admitted in CCU were selected by consecutive sampling. Written informed consent was obtained from the study subjects after explanation.

\section{Study Procedure}

The patients who got admitted in the Department of Cardiology, according to the inclusion and exclusion criteria were selected as study population. Detailed history, physical examination, routine laboratory examinations and echocardiography were done. The diagnosis of heart failure was based on the criteria recommended by the 2021 ACC / AHA HF guidelines. Total of 45 patients were included in this study. All participants were interviewed meticulously. Before interviewing of the patient, purpose and procedure of the study were discussed with the patients. After getting consent, the patients were asked few questions in different subheading including limitations of daily physical activities to classify NYHA class III \& IV, duration of $\mathrm{HF}$ and prior hospotalization, potential risk factors of aggravation of HF, co-morbid disease. At emergency department patients were treated according to heart failure guidelines and venous blood samples were collected for haematological and biochemical analysis. The bed side echocardiogram was performed using $G E 9$ machine to all eligible patients at admission. Radiological evaluation was done. These subjects were classified into NYHA class III \& IV of stage C and D HF. The patients were observed during mean hospital stay of 9 days and physical examination and the transition of NYHA class III \& IV to NYHA class I/II/III was noted before discharge. The clinician- assessed questionnaire for NYHA class of HF was used to evaluate the transition of higher NYHA class to lower NYHA class after guideline directed medical therapy for $\mathrm{HF}$.

\section{Statistical analysis}

After editing data analysis was carried out by using the Statistical Package for Social Science (SPSS) version 25.0 windows software. Categorical data were expressed as frequency and percentages. Continuous data were expressed as mean $\pm \mathrm{SD}$. Association between categorical data were analyzed by Chi-Square test. Continuous data were compared by Student's t-test. Independent t-test was used to compare a quantitative variable between two groups. Paired t-test was used to compare quantitative variables on admission and discharge as well as Wilcoxon's Signed Rank test was used to compare the qualitative variables on admission and discharge. Multivariable Cox Proportional Hazard Regression analysis was used to determine association between variables and mortality. $\mathrm{P}$ value of less than 0.05 was considered significant.

\section{Results}

A total of 45 patients were selected for the study. Among them, 11 patients classified to NYHA class III \& 22 cases belonged to NYHA class IV of stage C HF. Similarly, 5 patients were in NYHA class III \& 7 patients were in NYHA class IV of stage D HF.

\section{Demographics}

The mean age \pm SD for NYHA class III and IV of stage C and D HF were $62.5 \pm 9.8,60.6 \pm 13.4,54.4 \pm 15.8$ and $54 \pm 19.8$ successively. Majority of subjects were $>60$ years for stage $\mathrm{C}$ and $>50$ years for stage $\mathrm{D}$ HF. Both the NYHA class of Stages C \&D HF had male predominance. Most of the subjects had normal BMI (Table 1).

Table I

Demographic profile of Stage C and D HF study subjects $(n=45)$

\begin{tabular}{lcccc}
\hline$<40$ & $0(0)$ & $2(9.1)$ & $1(16.7)$ & $3(42.9)$ \\
$41-50$ & $1(9.1)$ & $2(9.1)$ & $0(0)$ & $0(0)$ \\
$51-60$ & $2(18.2)$ & $4(18.2)$ & $1(9.1)$ & $1(14.3)$ \\
$>60$ & $8(72.7)$ & $14(63.6)$ & $3(50)$ & $3(42.9)$ \\
Mean age \pm SD & $62.5 \pm 9.8$ & $60.6 \pm 13.4$ & $54.5 \pm 15.8$ & $54 \pm 19.8$ \\
Gender & & & & \\
Male & $8(72.7)$ & $11(50.0)$ & $3(60)$ & $7(100)$ \\
Female & $3(27.3)$ & $11(50.0)$ & $2(40)$ & $0(0)$ \\
\hline
\end{tabular}




\section{Primary cause of Stage C \& D HF:}

The primary cause for Chronic HF for stage C and D. Ischemic cardiomyopathy $28(84 \%)$ vs $10(83.3 \%)$ followed by valvular heart disease $5(15 \%)$ vs $1(8.3 \%)$ and for dilated cardiomyopathy were $3(9 \%)$ vs $1(8.3 \%)$ for stage C \& D HF respectively (Figure 1).

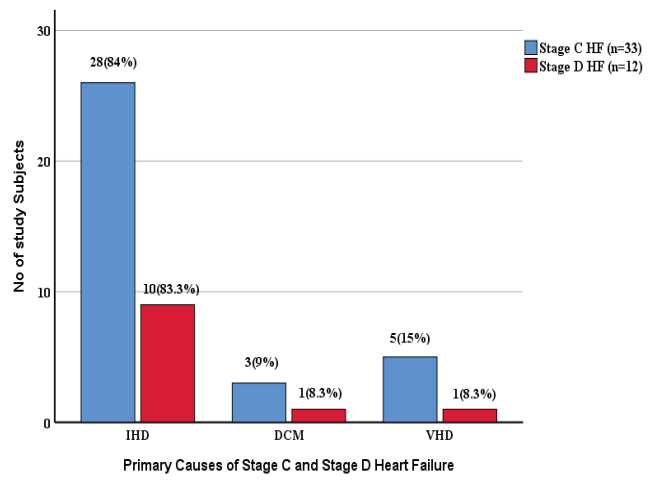

Figure 1: Primary cause of Stage C and D HF in $(\mathrm{n}=45)$
Clinical presentation of Stage C and D HF subjects on admission and discharge

Table II (a) \& (b) shows clinical presentation of NYHA class III \& IV HF were noted on admission and discharge and difference was found to be statistically significant for most of the presentations.

Dyspnea on exertion for stage C HF and dyspnea on rest for stage D HF subjects showed statistically significant difference $(p<0.001) \&(p<0.046)$. Crepitation was found in all the study subjects of both the stages of HF on admission but it disappeared on discharge and the difference was statistically significant with $\mathrm{p}<0.001 \&$ $\mathrm{p}<0.008$. The difference between pulse rate $(\mathrm{p}<0.001 \&$ $\mathrm{p}<0.002)$, SBP $(\mathrm{p}<0.003 \& \mathrm{p}<0.000)$, DBP $(\mathrm{p}<0.002 \&$ $\mathrm{p}<0.43)$ and respiratory rate $(\mathrm{p}<0.001 \& \mathrm{p}<0.82)$ for stage $\mathrm{C}$ and $\mathrm{D} H \mathrm{HF}$ found to be statistically significant except for the DBP and respiratory rate for stage D subjects which could not achieve statistical significance.

Table II (a)

Clinical presentation of Stage C HF subjects on admission \& discharge

\begin{tabular}{|c|c|c|c|}
\hline Variables & On Admission $(\mathrm{n}=33)$ & On Discharge $(\mathrm{n}=27)$ & p-value \\
\hline Dyspnea & $33(100)$ & $3(11.1)$ & ${ }^{\mathrm{s}} 0.001 *$ \\
\hline Raised JVP & $33(100)$ & $6(22.2)$ & ${ }^{\mathrm{s}} 0.001 *$ \\
\hline Crepitation & $33(100)$ & $5(18.5)$ & ${ }^{\mathrm{s}} 0.001 *$ \\
\hline Leg Edema & $33(100)$ & $9(33.3)$ & ${ }^{\mathrm{s}} 0.001 *$ \\
\hline Anaemia & $28(84.8)$ & $7(25.9)$ & ${ }^{\mathrm{s}} 0.021 *$ \\
\hline Pulse (per min) & $101.8 \pm 15.8$ & $73 \pm 29.7$ & ${ }^{\mathrm{s}} 0.001 \mathrm{Q}$ \\
\hline $\mathrm{SBP}(\mathrm{mm}$ of $\mathrm{Hg})$ & $110.5 \pm 26.5$ & $93.7 \pm 13.8$ & ${ }^{\mathrm{s}} 0.003 \mathrm{Q}$ \\
\hline $\mathrm{DBP}(\mathrm{mm}$ of $\mathrm{Hg})$ & $93.3 \pm 38.5$ & $61.8 \pm 24.2$ & ${ }^{\mathrm{s}} 0.002 \mathrm{Q}$ \\
\hline RR (breaths/min) & $25.5 \pm 5.5$ & $14.3 \pm 5.3$ & ${ }^{\mathrm{s}} 0.001 \mathrm{Q}$ \\
\hline
\end{tabular}

Table II (b)

Clinical presentation of Stage D HF subjects on admission \& discharge

\begin{tabular}{lccc}
\hline Variables & On Admission $(\mathrm{n}=12)$ & On Discharge $(\mathrm{n}=8)$ & $\mathrm{p}$-value \\
\hline Dyspnea & $12(100)$ & $2(25)$ & ${ }^{\mathrm{s}} 0.046^{*}$ \\
Raised JVP & $12(100)$ & $1(12.5)$ & ${ }^{\mathrm{s}} 0.046^{*}$ \\
Crepitation & $12(100)$ & $1(12.5)$ & ${ }^{\mathrm{s}} 0.008^{*}$ \\
Leg Edema & $12(100)$ & $2(25)$ & ${ }^{\mathrm{s}} 0.025^{*}$ \\
Anaemia & $11(84.6)$ & $4(50)$ & ${ }^{\mathrm{ns}} 0.317^{*}$ \\
Pulse (per min) & $97.7 \pm 21.2$ & $70.7 \pm 12$ & ${ }^{\mathrm{s}} 0.002 \mathrm{Q}$ \\
SBP(mm of Hg) & $105.7 \pm 23$ & $107.1 \pm 9.5$ & ${ }^{\mathrm{s}} 0.000 \mathrm{Q}$ \\
DBP(mm of Hg) & $62.9 \pm 15$ & $74.3 \pm 9.8$ & $\mathrm{~ns} 0.439 \mathrm{Q}$ \\
RR (breaths/min) & $28 \pm 5.7$ & $12.8 \pm 5.3$ & $\mathrm{~ns} 0.829 \mathrm{Q}$ \\
\hline
\end{tabular}


Frequency of Transition of patients with NYHA class III / IV to NYHA class I / II / III

Table III (a) \& (b)

Hematological, biochemical profile and Echocardiographic findings of NYHA class III \& IV of Stage C\& D HF study subjects on admission

\begin{tabular}{|c|c|c|c|}
\hline Variables & NYHA III (n=11) & NYHA IV $(n=22)$ & $\mathrm{p}$-value \\
\hline $\mathrm{Hb}(\mathrm{g} / \mathrm{dl})$ & $11 \pm 2.2$ & $10.5 \pm 2.6$ & ${ }^{\mathrm{ns}} 0.599$ \\
\hline S. Creatinine $(\mathrm{mg} / \mathrm{dl})$ & $1.5 \pm 0.4$ & $2.4 \pm 1.9$ & ${ }^{\mathrm{s}} 0.039$ \\
\hline $\mathrm{S} . \mathrm{Na}(\mathrm{mmol} / \mathrm{L})$ & $134.4 \pm 3$ & $133.6 \pm 6.4$ & ${ }^{\mathrm{ns}} 0.716$ \\
\hline Urea (mg/dl) & $45.3 \pm 1.56$ & $56.4 \pm 3.8$ & ${ }^{\mathrm{s}} 0.001$ \\
\hline NTproBNP pg/ml & $5223.7 \pm 5333.3$ & $11828.4 \pm 1652.7$ & ${ }^{\mathrm{ns}} 0.421$ \\
\hline LVEF (\%) & $34 \pm 5.7$ & $36.6 \pm 8.4$ & ${ }^{\mathrm{ns}} 0.385$ \\
\hline Variables & NYHA III $(n=5)$ & NYHA IV $(\mathrm{n}=7)$ & $\mathrm{p}$-value \\
\hline $\mathrm{Hb}(\mathrm{g} / \mathrm{dl})$ & $10.4 \pm 2.5$ & $9.1 \pm 2$ & ${ }^{\mathrm{ns}} 0.361$ \\
\hline S. creatinine $(\mathrm{mg} / \mathrm{dl})$ & $2.2 \pm 1.2$ & $6.2 \pm 0.7$ & ${ }^{\mathrm{s}} 0.035$ \\
\hline S. Na (mmol/L) & $134.4 \pm 5.1$ & $136 \pm 4.5$ & ${ }^{\mathrm{ns}} 0.594$ \\
\hline Urea (mg/dl) & $52.8 \pm 1.9$ & $66.6 \pm 6.3$ & ${ }^{\mathrm{s}} 0.007$ \\
\hline NTproBNP (pg/ml) & $10159 \pm 3575.2$ & $16196.3 \pm 1893.8$ & ${ }^{\mathrm{s}} 0.016$ \\
\hline LVEF (\%) & $27.3 \pm 3.5$ & $21.8 \pm 4.7$ & ${ }^{\mathrm{s}} 0.043$ \\
\hline
\end{tabular}

Hematological, biochemical profile and Echocardiographic findings of NYHA class III \& IV of Stage C \& D HF study subjects on admission

Table III (a) and (b) displays the contrast value of haematological, biochemical and echocardiographic variables in NYHA class III \& IV of stage C \& D respectively. None of the variables among NYHA class III \& IV of stage C HF subjects as haemoglobin ( $11 \pm 2.2$ vs $10.5 \pm 2.6, \mathrm{p}<0.59)$, serum sodium $(134.4 \pm 3$ vs $133.6 \pm 6.4, \mathrm{p}<0.71)$, NTproBNP $(5223.7 \pm 5333.3$ vs $11828.4 \pm 1652.7, \mathrm{p}=0.42)$ and $\mathrm{LVEF}(34 \pm 5.7$ vs $36.6 \pm 8.4$, $\mathrm{p}<0.38$ ) showed any statistically significant difference between the two classes except serum creatinine and urea level which was statistically significant with $\mathrm{p}$ value $<0.039$ $\&<0.001$ respectively.

Radiological finding of NYHA class III \& IV of Stage C \& D HF

Table IV (a) \& (b) displays that majority of subjects of NYHA class III / IV of stage C \& D HF presented with pulmonary congestion and cardiomegaly radiographically which was higher in frequency and statistically significant mainly for NYHA class IV patients of both stage C and D HF.

Table IV (a) \& (b)

Radiological finding of NYHA class III \& IV of Stage C \& D HF

\begin{tabular}{lccc}
\hline Variables & $\begin{array}{c}\text { NYHA class III } \\
(\mathrm{n}=11)\end{array}$ & $\begin{array}{c}\text { NYHA class IV } \\
(\mathrm{n}=22)\end{array}$ & $\mathrm{p}$-value \\
\hline Pulmonary Congestion & $10(76.9)$ & $22(100.0)$ & ${ }^{\mathrm{s}} 0.021$ \\
Cardiothoracic Ratio $>0.5$ & $9(69.2)$ & $22(100.0)$ & ${ }^{\mathrm{s}} 0.007$ \\
\hline Variables & NYHA class III & NYHA class IV & $\mathrm{p}$-value \\
& $(\mathrm{n}=5)$ & $(\mathrm{n}=7)$ & \\
\hline Pulmonary Congestion & $3(50.0)$ & $7(100.0)$ & ${ }^{\mathrm{s}} 0.033$ \\
Cardiothoracic Ratio & $2(33.3)$ & $7(100.0)$ & ${ }^{\mathrm{s}} 0.009$ \\
\hline
\end{tabular}


Transition of NYHA class III to NYHA class I / II of Stage C HF 11 patients with NYHA class III of Stage C HF were admitted with a mean hospital stay of 9 days, $8(73 \%)$ subjects were upgraded to NYHA class I, 2 (18\%) subjects to NYHA class II, 1 (9\%) mortality (Figure 2)

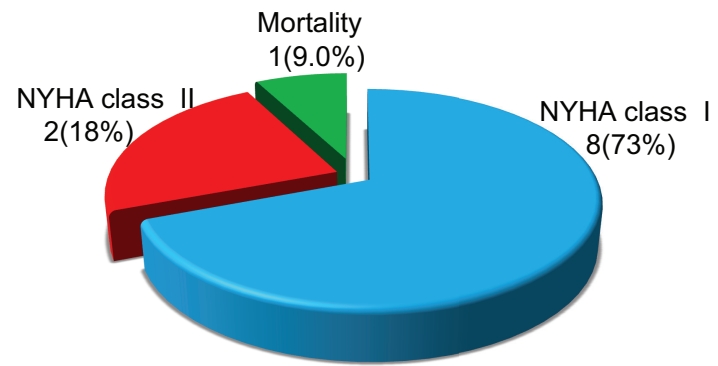

Fig.-2: Transition of NYHA class III to NYHA class I / II of Stage $\mathrm{CHF}$

\section{Transition of NYHA class IV to NYHA class I / II / III of Stage C HF}

22 admitted patient with NYHA class IV of Stage C HF after treated with group of heart failure drugs with average range of mean hospital stay of 9 days, 11 (49\%) cases were upgraded to NYHA class I, $4(20 \%)$ subjects to NYHA class II, 2 (9\%) subjects to NYHA class III, 5 (22\%) mortality (Figure 3)

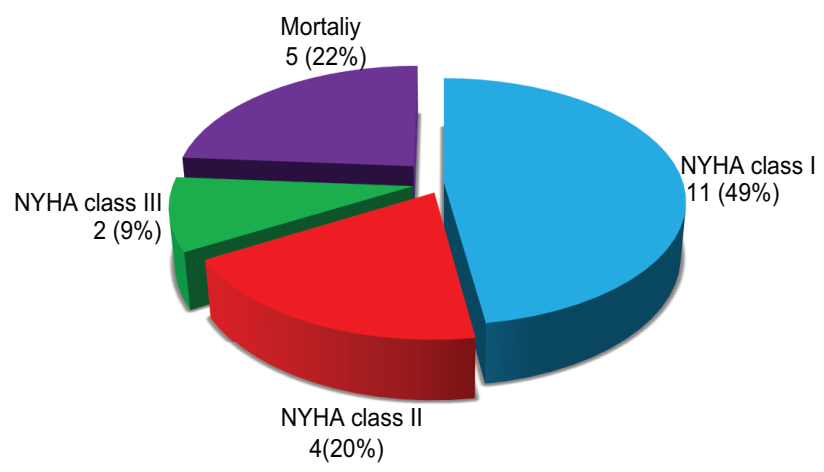

Fig.-3: Transition of NYHA class IV to NYHA class I / II / III of Stage C HF

\section{Transition of NYHA class III to NYHA class II / III of Stage D HF}

Out of 5 cases in NYHA class III of Stage D HF who were admitted for mean hospital stay of 9 days and treated with heart failure drugs, among them NYHA class III of Stage D HF, none (0\%) subject was upgraded to NYHA class I, $3(60 \%)$ subject to NYHA class II, $1(20 \%)$ subject remained in class III, 1 (20\%) mortality (Figure 4).

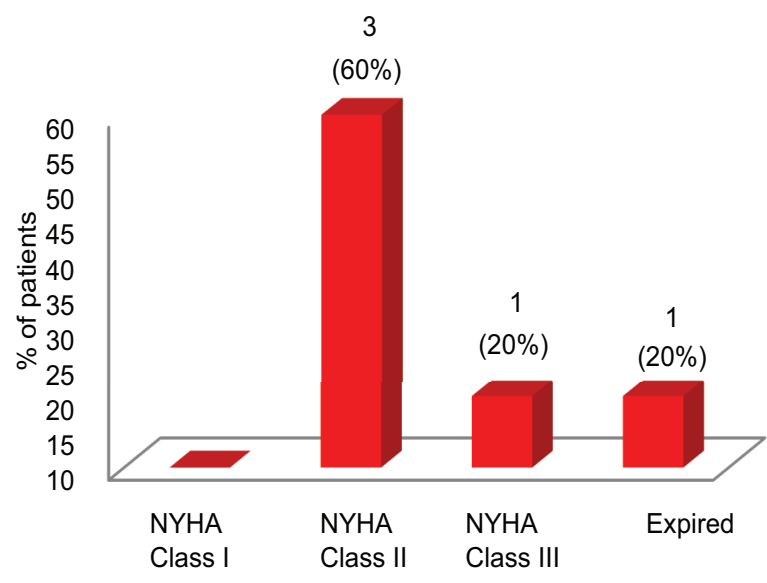

Fig-4: Transition of NYHA class III to NYHA class II / III of Stage D HF

\section{Transition of NYHA class IV to NYHA class II / III of Stage D HF}

7 patients with NYHA class IV of Stage D HF were admitted for mean of 9 days and were treated with HF medications, the following outcome were noted: NYHA class IV of Stage D HF, no subject was upgraded to NYHA class I, 3 (43\%) subjects to NYHA class II, 1 (14\%) subject to NYHA class III, 3 (43\%) mortality (Figure 5).

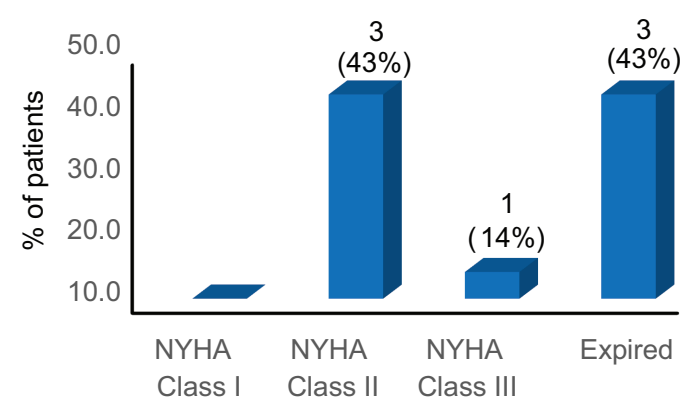

Fig.-5: Transition of NYHA class IV to NYHA class II / III of Stage D HF

\section{Hazard Ratio for all-cause mortality for stage C \& D HF subjects}

The hazard ratio of 45 subjects who were being observed over mean hospital stay of 9 days admitted for Acute on chronic HF, 10 subjects expired. The table displays the effect of variables on mortality. The hazard ratio for male in comparison to female is 2.42 , age $>60$ years is 2.52 , $\mathrm{DM}$ is 1.83 , $\mathrm{LVEF}<30 \%$ is 1.97 , CKD is 1.89 , NYHA class IV is 4.82 (Table V). 
Table V

Hazard Ratio for all-cause mortality for stage $C \& D$ HF subjects

\begin{tabular}{|c|c|c|c|c|}
\hline \multicolumn{3}{|c|}{ Variables } & \multicolumn{2}{|l|}{ Hazard Ratio } \\
\hline Male & & & 2.42 & $1.54-3.62$ \\
\hline Age (>60 years) & ! 1 & & 2.52 & $1.56-3.01$ \\
\hline Over weight & - & & 2.99 & $2.48-3.66$ \\
\hline $\begin{array}{r}\text { DM } \\
\text { JVEF }\end{array}$ & $\bullet \quad 1$ & & 1.83 & $1.23-3.25$ \\
\hline & 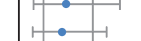 & & 1.97 & $1.29-3.06$ \\
\hline NYHA class III & .5 & 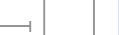 & 1.89 & $1.28-2.78$ \\
\hline NYHA Class IV & & . & 2.06 & $1.57-4.21$ \\
\hline Cardiothoracic Ratio >0.5 & $\mapsto$\begin{tabular}{l|l}
$\mapsto$ \\
$\longmapsto$
\end{tabular} & & 4.82 & $3.59-5.38$ \\
\hline 0.5 & $\begin{array}{lll}1.5 & 2.5 & 3\end{array}$ & $\begin{array}{lll}5 & 4.5 & 5.5\end{array}$ & 1.85 & $1.77-3.17$ \\
\hline
\end{tabular}

\section{Discussion}

The mean age of the patients of this study for NYHA class III/IV of stage C HF were consistent with DIG $^{18}$ and SOLVD $^{17}$ trial where it was found to be 63 and 60 years respectively but specific age for NYHA functional class and stages of HF was not mentioned. A retrospective study of DIG trial performed by Ahmed et al. 2006, ${ }^{3}$ demonstrated the mean age for NYHA class III \& IV were 71 years. Increased occurrence of HF in younger age as compared to western counterparts may be explained by increased prevalence of diabetes, dyslipidemia and CAD in younger population in this part of the world. The CARDIA $^{19}$ study also observed that HF before 50 years of age was more common among blacks and higher blood pressure, higher body-mass index, fewer years of education, lower HDL cholesterol, the presence of chronic kidney disease, and the presence of diabetes were associated with the subsequent development of heart failure. ${ }^{7}$ The mean age of NYHA class III / IV of Stage D $\mathrm{HF}$ in this study was found to be 54 years which was lower than NYHA class III / IV of Stage C HF that could be because of smaller study subjects in Stage D HF and the etiologies were mainly DCM and IHD which is more pronounced in younger age.

Pillai and Ganapathi ${ }^{5}$ reported in 2013 the comparison age of the HF patients in this region and western populations. Patients admitted with HF in South Asia region are relatively younger than their western counterparts. The primary causes of HF in majority of the NYHA class III / IV of Stage C and D HF subjects were IHD followed by Rheumatic Heart Disease and DCM which is similar to retrospective study of DIG trial. ${ }^{3}$ In a small study of 125 patients from India, Rheumatic Heart Disease was the commonest heart disease $(52.8 \%)$ followed by IHD and / or hypertensive heart disease $(27 \%){ }^{13}$
In a study from Pakistan of 196 patients (2007), 77\% of HF was due to IHD. ${ }^{8}$ In Bangladesh, a retrospective study was done at a tertiary hospital and found that majority (35.79\%) had CAD as the principal etiological factor, whereas hypertension was the primary risk factor for HF in $29.14 \%$ of cases. ${ }^{9}$ As expected, hypertension is the major risk factor found in this study ranging from $50-70 \%$ of study subjects in NYHA class III / IV of Stage C and D HF which is supported by similar finding in retrospective DIG trial ${ }^{3}$. SOLVD ${ }^{17}$ and DIG $^{18}$ trial also found the similar result for HTN in $42 \%$ and $45 \%$ subjects respectively.

DM was present in about $45-71 \%$ of the patients as in contrast to SOLVD and DIG trial where it was found in $24 \%$ and $28 \%$ respectively. DM is one of the most common association with CAD and majority of the study population had Ischemic cardiomyopathy and this could be the reason for high prevalence of DM as a consequence of unhealthy lifestyle maintained in these subjects. CKD was the most pronounced comorbidity found in this study which was statistically significant and higher in frequency. particularly in NYHA class IV of stage C and D HF subjects. It was found to be present in $71 \%$ of the subjects in the present study whereas found in $58 \%$ subjects. ${ }^{3}$

Dyspnea on exertion $(100 \%)$ and at rest (100\%) was the main presenting feature in NYHA class III and IV of stage $\mathrm{C}$ and D HF. ${ }^{10}$ Crepitation (100\%), leg edema (100\%) followed by raised JVP (100\%) were the next manifestation in both the NYHA classes and it is similarly related to the results from different study. ${ }^{10}$ The proportion of the clinical presentation were decreased on discharge but it's difficult to compare to other studies as there is scarcity of data regarding these findings on discharge. The SBP and DBP were similar in the present study. ${ }^{3,10}$ Pulse rate was higher on admission and normal at discharge in the present study. Higher pulse rate is a marker of greater neurohormonal activation. The SHIFT ${ }^{14}$ study showed that heart rate is important in pathophysiology of HF and heart rate reduction per se is mechanism responsible for improvement in clinical outcome.

Pulmonary congestion at chest X-ray is a proven independent prognostic determinant of mortality. The radiological finding revealed pulmonary congestion $(100 \%)$ and cardiomegaly $(100 \%)$ in this study for stage $\mathrm{C}$ and D HF but it was at lower side in another study conducted.

Raised BNP level above the reference limit indicated that subjects of this study were in decompensated stage of HF in this study. PROTECT ${ }^{15}$ study reported that NTproBNP levels serve as non-invasive window for the physiological 
state of cardiac performance and remodeling and concentration of NTproBNP are strongly associated with outcome in chronic HF. Higher level was observed in higher NYHA class of Stage D HF patients. This was in accordance with the findings of OPTIMIZE HF registry. ${ }^{11}$

A statistically significant difference of LVEF has been found between NYHA class III and IV of stage D HF subjects in this study which ranges from $34-36 \%$. The lower EF was observed with higher NYHA class mainly with Stage D HF patients where it was found to be in a range from $21-27 \%$.

A study revealed that mortality was four fold higher with reduced EF. ${ }^{16}$ Higher level was observed in higher NYHA class of Stage D HF patients. This was in accordance with the findings of OPTIMIZE HF registry. ${ }^{11}$ This study has sub-classified the NYHA class III / IV of stage C and D HF patients separately to find out the frequency of transition of NYHA class in the same stage of HF. This study has found that NYHA class III of stage C HF patients entered to NYHA class I (73\%), class II $(18 \%)$ and $9 \%$ expired. The NYHA class IV patients of Stage C got transformed to class I (49\%), class II (20\%), class III (9\%) and 22\% got expired. Similarly, the NYHA class III of Stage D HF patients shifted to class II (60\%), class III (20\%) and 20\% expired. None were shifted to NYHA class I.

This study also tried to find out the proportion on NYHA class IV of Stage D HF patients which were considered the worst patients amongst all the classes, but the study revealed $43 \%$ got transited to class II, $14 \%$ to class III and $43 \%$ expired. Ahmed A.et al., $2006^{3}$ reported mortality rate to be $35 \%$ for NYHA class III and $58 \%$ for NYHA class IV (the particular stage of HF has not been mentioned) in contrast to this study where frequency of mortality rate is lower and it could be because of lower age with less comorbidities and less presentation to hospital in this region. Several factors account for the poor outcome like the higher age, male gender, higher NYHA class, underweight, high number of comorbidities like HTN, DM, CKD, COPD, no optimization of the drugs with poor compliance, $\mathrm{EF}<30 \%$, high $\mathrm{BNP}$ level, lower S. sodium, high urea level, HR $>100 \mathrm{~b} / \mathrm{m}$, SBP $<100$ $\mathrm{mmHg}$, recent hospital stay or repeated hospitalizations, haemodynamic profile, cardiomegaly, S3, poor quality of life, crepitations, edema, raised JVP, hepatomegaly, ascites, smoking, anaemia, diuretic resistance ${ }^{6}$. The mortality rate of NYHA class IV is 8 times higher than the lower NYHA classes. ${ }^{3}$

\section{Conclusion}

This study demonstrated that the transition of NYHA class III to NYHA class I / II of stage C and D HF is higher in frequency and statistically significant. So it has made possible for the physicians to address these patients for whom optimal medical therapy can be used vigorously and the prognosis of these low risk patients may be improved further. The study has also found that the transition of NYHA class IV to NYHA class I / II / III of stage $\mathrm{C}$ and D HF is higher in frequency but not significant statistically. Thus it has recognized these high risk patients for morality and help to tailor the management. These patients are the ideal candidate for transplant or long term Mechanical Circulatory Support either as bridge to transplant or Destination therapy. As we belong to resource constraint setting, accessibility or affordability is major challenge. So the management plan for these high risk patients should be palliative or hospice care.

\section{Limitation \\ Sample size \\ Observation for short period \\ NYHA has poor reproducibility}

\section{Conflict of interest}

Authors declare no conflict of interest.

\section{References}

1. Bilbao A, Escobar A, García-Perez L, Navarro G, Quirós R. The Minnesota living with heart failure questionnaire: comparison of different factor structures. Health and quality of life outcomes. 2016;14(1):1-1.

2. Holland, R., Rechel, B., Stepien, K., Harvey, I. and Brooksby, I., . Patients' self-assessed functional status in heart failure by New York Heart Association class: a prognostic predictor of hospitalizations, quality of life and death. Journal of cardiac failure, 2010;16(2):150-56.

3. Ahmed, A., Aronow, W.S. and Fleg, J.L. Higher New York Heart Association classes and increased mortality and hospitalization in patients with heart failure and preserved left ventricular function. American heart journal, 2006;151(2): 444-50.

4. Caraballo, C., Desai, N.R., Mulder, H., Alhanti, B., Wilson, F.P., Fiuzat, M., Felker, G.M., Piña, I.L., O’Connor, C.M., Lindenfeld, J. and Januzzi, J.L. Clinical implications of the New York heart association classification. Journal of the American Heart Association, 2019;8(23):p.e014240.

5. Harikrishnan Sivadasan Pillai and Sanjay Ganapathi; Department of Cardiology, Sree Chitra Tirunal Institute for Medical Sciences and Technology, Trivandrum, Kerala, India. Current Cardiology Reviews, 2013;9:102-111;Heart Failure in South Asia.

6. Crespo Leiro, M.G., Metra, M., Lund, L.H., Milicic, D., Costanzo, M.R., Filippatos, G., Gustafsson, F., Tsui, S., Barge Caballero, E., De Jonge, N. and Frigerio, M.. Advanced heart failure: a position statement of the Heart Failure Association of the European Society of Cardiology. European journal of heart failure, 2018;20(11), 1505-35. 
7. Bibbins-Domingo, K., Pletcher, M.J., Lin, F., Vittinghoff, E., Gardin, J.M., Arynchyn, A., Lewis, C.E., Williams, O.D. and Hulley, S.B. Racial differences in incident heart failure among young adults. New England Journal of Medicine, 2009;360(12): 1179-90.

8. Jafary, F.H., Kumar, M. and Chandna, I.E. Prognosis of hospitalized new-onset systolic heart failure in Indo-Asians-a lethal problem. Journal of cardiac failure, 2007;13(10):855-60

9. Islam, A. M., Mohibullah, A. K. M. and Paul, T. 'Cardiovascular disease in Bangladesh: a review.' Bangladesh Heart Journal, 2016;31(2):80-99.

10. Muntwyler, J., Abetel, G., Gruner, C. and Follath, F. One-year mortality among unselected outpatients with heart failure. European heart journal, 2002;23(23):1861-66.

11. Yancy, C. W., Lopatin, M., Stevenson, L. W., De Marco, T., Fonarow, G. C. and ADHERE Scientific Advisory Committee and Investigators 'Clinical presentation, management, and in-hospital outcomes of patients admitted with acute decompensated heart failure with preserved systolic function: a report from the Acute Decompensated Heart Failure National Registry (ADHERE) Database.' Journal of the American College of Cardiology, 2007;47(1):76-84.

12. Vasan, R.S., Larson, ScD, M.G., Benjamin, E.J., Evans, J.C., Reiss, C.K. and Levy, D. Congestive heart failure in subjects with normal versus reduced left ventricular ejection fraction: prevalence and mortality in a population-based cohort. Journal of the American College of Cardiology, 1999;33(7):1948-55.

13. Joshi, P.P., Mohanan, C.J., Sengupta, S.P. and Salkar, R.G.. Factors precipitating congestive heart failure-role of patient noncompliance. The Journal of the Association of Physicians of India, 1999;47(3):294-95.

14. Böhm M, Swedberg K, Komajda M, Borer JS, Ford I, DubostBrama A, Lerebours G, Tavazzi L, SHIFT investigators. Heart rate as a risk factor in chronic heart failure (SHIFT): the association between heart rate and outcomes in a randomised placebocontrolled trial. The Lancet. 2010;376(9744):886-94.

15. Weiner, R.B., Baggish, A.L., Chen Tournoux, A., Marshall, J.E., Gaggin, H.K., Bhardwaj, A., Mohammed, A.A., Rehman, S.U., Barajas, L., Barajas, J. and Gregory, S.A. Improvement in structural and functional echocardiographic parameters during chronic heart failure therapy guided by natriuretic peptides: mechanistic insights from the ProBNP Outpatient Tailored Chronic Heart Failure (PROTECT) study. European journal of heart failure, 2013;15(3):342-51.

16. Vasan, R.S., Larson, ScD, M.G., Benjamin, E.J., Evans, J.C., Reiss, C.K. and Levy, D. Congestive heart failure in subjects with normal versus reduced left ventricular ejection fraction: prevalence and mortality in a population-based cohort. Journal of the American College of Cardiology, 1999;33(7):1948-55.

17. Vermes E, Tardif JC, Bourassa MG, Racine N, Levesque S, White M, Guerra PG, Ducharme A. Enalapril decreases the incidence of atrial fibrillation in patients with left ventricular dysfunction: insight from the Studies Of Left Ventricular Dysfunction (SOLVD) trials. Circulation. 2003;107(23):2926-31.

18. Aguirre Dávila L, Weber K, Bavendiek U, Bauersachs J, Wittes J, Yusuf S, Koch A. Digoxin-mortality: randomized vs. observational comparison in the DIG trial. European heart journal. 2019;40 (40):3336-41.

19. Pereira MA, Kartashov AI, Ebbeling CB, Van Horn L, Slattery ML, Jacobs Jr DR, Ludwig DS. Fast-food habits, weight gain, and insulin resistance (the CARDIA study): 15-year prospective analysis. The lancet. 2005;365(9453):36-42. 